Stiftung für Technologiefolgen-Abschätzung Fondation pour l'évaluation des choix technologiques Fondazione per la valutazione delle scelte tecnologiche Foundation for Technology Assessment


Ursula Meidert, Mandy Scheermesser, Yvonne Prieur, Stefan Hegyi, Kurt Stockinger, Gabriel Eyyi, Michaela Evers-Wölk, Mattis Jacobs, Britta Oertel, Heidrun Becker

\title{
Quantified Self - Schnittstelle zwischen Lifestyle und Medizin
}




\section{Ethische Aspekte}

In diesem Kapitel wird diskutiert, welche Werte durch QS berührt werden und möglicherweise negative Auswirkungen für Individuen und Gesellschaft haben können. Wie bereits im vorangegangenen Kapitel Recht (siehe Kapitel 9) erwähnt, werden die Themenbereiche Recht und Ethik im Kontext von QS separat diskutiert, obwohl sich in beiden Themenbereichen ähnliche Fragen stellen. Entsprechend werden in diesem Kapitel die Ergebnisse der Literaturanalyse und der Experteninterviews aus ethischer Sicht dargestellt. Es werden ethische Aspekte und Normen skizziert, die von QS sowie den zugehörigen Big-Data-Anwendungen tangiert sind.

Im Weiteren stellt sich in der ethischen Diskussion die Frage nach dem „Wie wollen wir leben?". Im Kontext des Trends QS soll gemäss Peter Dabrock auch danach gefragt werden, worauf man sich bei diesem Trend einlässt (Dabrock, Deutscher Ethikrat, 2015).

\subsection{Ausgangslage und Fragestellung aus ethischer Sicht}

Technikgestütztes Messen ist - zumindest in der Medizin - kein neues Phänomen (vgl. Kapitel 4.1 und 6.1). Der Einsatz digitaler Technologie weist jedoch einige Besonderheiten auf, beispielsweise den Umfang und die Ubiquität (das Nichtgebundensein an einen Standort) der erhobenen Daten (Fangerau, Grimmert \& Albrecht, 2016). In zunehmend einfacherer Weise können diese Daten anschliessend gespeichert und ausgewertet werden (Hauser et al., 2017). Diese Entwicklung nährt Hoffnungen, schürt aber auch Ängste. So sehen manche die Datenflut als einen Innovationstreiber. Es wird beispielsweise nicht mehr nur von „Personalized Medicine“ (siehe Kapitel 6.7), sondern bereits von „Precision Medicine“ gesprochen, deren wichtigste Treiber digitale Tools sind, die ein Echtzeit-Monitoring von Patientinnen und Patienten (sogenannte Feedback Loops) ermöglichen. Diese so erhobenen Patientendaten werden mit Daten aus klinischen Tests, Genanalysen und Algorithmen kombiniert, um damit für künftige Patientinnen und Patienten „präzisere und kostengünstigere medizinische Lösungen und Therapien" (Streiff \& Brenzikofer, 14.10.2016, S. 8) entwickeln zu können. Die Entwicklung, Vernetzung 
und Auswertung medizinischer Daten könnten zur Steigerung der Lebensqualität beitragen, wenn beispielsweise die verloren gegangene Selbstständigkeit durch intelligente Prothesen zurückgewonnen werden kann (Peier, 2016). Darüber hinaus sollen im Konzept der „Personalized Health" nicht nur einzelne Patientinnen und Patienten, sondern die gesamte Bevölkerung aus den Erkenntnissen Nutzen ziehen, die aus der Kombination von Genomics-Daten, klinischen Daten aus der Gesundheitsversorgung und Self-Tracking-Gesundheitsdaten Einzelner hervorgehen (SAMW-Bulletin, 1/2016; Neff \& Nafus, 2016).

Darbrock und Woopen (Deutscher Ethikrat, 2015) hingegen weisen auf die Bedrohung für Werte wie Freiheit und Privatsphäre hin oder gar auf die Bedrohung für das Recht auf informationelle Selbstbestimmung (Baeriswyl, 2016). Diese Ambivalenz verdeutlicht, dass im Zusammenhang mit Big Data (vgl. Kapitel 7.8) und QS diverse ethische Aspekte zu diskutieren sind. Hauser et al. (2017) fragen sich beispielsweise, welche Bedeutung Grundwerte wie Selbstbestimmung, Solidarität und Privatheit in einer datengetriebenen Welt haben, ob gewisse Daten ein öffentliches Gut darstellen und ob wir unter Umständen verpflichtet sind, gewisse Daten preiszugeben, um die Gesellschaft als Ganzes effizienter zu gestalten. Nach Peier (2016) ist der technische sowie medizinische Fortschritt ohnehin nicht aufzuhalten. Ethische und moralische Überlegungen sollten jedoch zu Regeln führen, die für "die Entwicklung von Computern, Robotern, Programmen und Algorithmen gelten" (S. 1428).

\subsection{Schnittstelle zwischen Lifestyle und Medizin}

Gemäss Brigitte Tag (persönliches Interview, 21.9.2016) werden viele Behandlungen und Therapien, die noch vor wenigen Jahren dem Bereich Lifestyle zugeordnet wurden, heute als medizinisch relevant betrachtet. Abgrenzungsfragen, d.h. Fragen danach, ob etwas dem Lifestyle- oder Medizinbereich zuzuordnen ist, würden daher künftig zunehmen. Tag betont auch, dass ,je mehr das Individuum und die Selbstwahrnehmung des Individuums in den Mittelpunkt der Wahrnehmung der Gesellschaft, des Rechts und der Ethik rückt, desto mehr werden Fragen des Lifestyles auch plötzlich zur Frage der Abweichung". Mit der Abweichung von der Norm kann eine an und für sich minimale Beeinträchtigung zu einem Krankheitsbild führen, welches dann tatsächlich eine medizinische Behandlung nach sich 
ziehen kann. ${ }^{24}$ Dies zeigt, dass die Grenzen zwischen Lifestyle und Medizin fliessend sind. Dennoch müssen Grenzen festgelegt werden, weil unser Gesundheitssystem einerseits an Grenzen der Belastbarkeit stösst, andererseits aber auch Begehrlichkeiten entstehen, die nicht durch die Gesellschaft getragen werden sollen (Tag, persönliches Interview, 21.9.2016).

\subsection{Diskussion ethischer Aspekte und Normen}

In der deutschen Studie "Chancen und Risiken von Gesundheits-Apps“ (CHARISMHA) zeigen Fangerau, Grimmert \& Albrecht (2016) mit Verweis auf diverse Studien auf, dass ethische Herausforderungen oft einseitig auf Risiken reduziert werden, wobei es durchaus auch Anwendungschancen gebe. Allgegenwärtig sei zudem die Diskussion betreffend Dilemmasituationen. Solche ergäben sich aus der Abwägung mitunter konkurrierender Interessen und Werte - beispielsweise "Transparenz" und „Privatheit" im Austausch von Gesundheitsinformationen oder „Autonomie" und „Kontrolle“ im Umgang mit der eigenen Gesundheit - und seien jeweils mit Risiken in beide Richtungen behaftet (Fangerau, Grimmert \& Albrecht, 2016). Nach der Autorengruppe um Abbas würden klassische Dilemmasituationen oft nur subjektive Lösungen zulassen (Abbas, Michael \& Michael, 2014, nach Fangerau, Grimmert \& Albrecht, 2016).

Nachfolgend werden ethische Aspekte und Normen skizziert, die von QS- sowie den zugehörigen Big-Data-Anwendungen tangiert sind.

\subsubsection{Privatheit bzw. Privatsphäre}

Da die Anwendungen von Big Data nicht nur aus anonymisierten, sondern weitgehend auch aus personenbezogenen Daten bestehen, birgt dies Gefahren für das individuelle Recht auf Privatsphäre. ${ }^{25}$ Nach Fischer et al. (2015) haben wir "[d]urch die Nutzung von kostenlosen Internetdiensten, sozialen Medien und Netzwerken

24 Beispielsweise kann die Abweichung von der Norm gerade bei jungen Menschen Auswirkungen auf die Psyche haben und sich nach und nach zu einem Krankheitsbild entwickeln (Tag, persönliches Interview, 21.9.2016).

25 Privatheit und Privatsphäre werden nachfolgend synonym verwendet. 
oder Kundenkarten [...] bereits einen grossen Teil unserer Privatsphäre preisgegeben“(S. 12). Auch Hussain et al. (2015, nach Fangerau, Grimmert \& Albrecht, 2016) äussern im Zusammenhang mit Gesundheits-Apps Bedenken bezüglich der Privatheit. Zwar mögen einzelne Merkmale nicht wirklich privat sein, doch die Kombination verschiedener Merkmale kann zu überwiegend privaten Rückschlüssen führen (Hauser et al., 2017; Fischer et al., 2015), vor allem dann, wenn Daten zu anderen Zwecken verwendet werden als den ursprünglich vorgesehenen (sogenannte Sekundärnutzung ${ }^{26}$; Hauser et al., 2017). Die sekundäre Verwendung von Daten spielt gerade bei Big-Data-Anwendungen eine besonders wichtige Rolle, denn die Kombination und Wiederverwendung von Daten bilden die wesentliche Grundlage dieser Technologie (Fischer et al., 2015) (siehe Kapitel 6).

Einen weiteren Aspekt bringt Brigitte Tag ein (persönliches Interview, 21.9.2016), wenn sie auf Situationen hinweist, in denen QS-Anwenderinnen und -Anwender Daten preisgeben, die einen engen Bezug zu Familienangehörigen aufweisen beispielsweise genetische Daten. Dies kann problematisch sein, wenn die Familienangehörigen weder darüber informiert noch um eine Einwilligung gebeten wurden. Hier stellt sich laut Tag (persönliches Interview, 21.9.2016) die Frage, ob eine solche Bekanntgabe überhaupt zulässig sei. Des Weiteren stellt sich die Frage nach der Verwendung der erhobenen Daten und der Rückwirkung im Lebenslängsschnitt: Was eine Person heute als richtig erachtet, erachtet sie in 20 Jahren vielleicht nicht mehr als richtig.

Auch eine Anonymisierung der Daten würde die Privatsphäre nicht genügend schützen, weil durch die Kombination verschiedener anonymisierter Datenbestände ein Rückschluss auf bestimmte Personen möglich wird (Tag, persönliches Interview, 21.9.2016; Hauser et al., 2017). Zudem besteht die Möglichkeit, dass Datensätze technisch deanonymisiert werden (Dabrock, Deutscher Ethikrat, 2015, siehe Kapitel 7.5). Für Hauser et al. (2017) ist nicht jeder Eingriff in die Privatsphäre in gleichem Masse problematisch. Es sei im Einzelfall und unter Abwägung der konkret involvierten Interessen zu entscheiden, ob ein Eingriff in die Privatsphäre letztlich erlaubt sei oder nicht (Hauser et al., 2017).

26 Definition Primär- und Sekundärnutzen von Daten, siehe Fischer et al., 2015, S. 15. 


\subsubsection{Transparenz}

Transparenz bildet die Voraussetzung für eine informierte Zustimmung zur Verwendung persönlicher Daten. Im Kontext von Big Data umfasst die Forderung nach Transparenz gemäss Hauser et al. (2017) das Recht jeder Person zu wissen, wer welche Daten über sie bearbeitet und wofür diese verwendet werden.

Sogenannte Datenschutzerklärungen sollen diesbezüglich Transparenz schaffen. Dabei handelt es sich jedoch meist um umfangreiche Texte, deren Kernaussagen für die Nutzenden nur schwer zu erfassen sind. Die Gründe hierfür sind mannigfaltig: Zum einen werden Daten in immer komplexeren Strukturen verarbeitet. Dies erschwert es zunehmend, die Nutzenden über die Einzelheiten der vorgesehenen Bearbeitungsvorgänge in einer klaren und einfachen Sprache aufzuklären. Zum anderen dienen Datenschutzerklärungen im Kern häufig weniger der schnellen Nutzerinformation, sondern vielmehr der rechtlichen Absicherung der Datenbearbeiter. Datenschutzerklärungen sollen Compliance demonstrieren und letztlich Angriffe in Form von rechtlichen Ansprüchen abwehren (Richter, 2017).

Die Transparenz nimmt eine wichtige Rolle bei der Gewinnung von Gesundheitsdaten ein (Data Mining). Die Autorinnen und Autoren des Grünbuchs über MobileHealth-Dienste (mHealth) der Europäischen Kommission sehen gerade an diesem Punkt ethische Bedenken. Problematisch ist ihrer Ansicht nach insbesondere die „Einhaltung des Grundsatzes der ausdrücklichen und in Kenntnis der Sachlage gegebenen Einwilligung, wo diese erforderlich ist" (S. 11) - beispielsweise wenn Patientinnen oder Patienten der Verwendung ihrer persönlichen Daten zu Forschungszwecken nicht ausdrücklich zugestimmt haben, obschon sie darum gebeten wurden (Europäische Kommission, 2014a). Im Weiteren verkaufen AppHersteller die von den Apps gesammelten Daten an Drittunternehmen, ohne die Nutzenden transparent zu informieren. Ein Grund mag gemäss Hauser et al. (2017) darin liegen, dass Unternehmen befürchten, die Kundinnen und Kunden würden ihre Dienste meiden, wenn die Big-Data-Praktiken transparent gemacht würden.

Im Rahmen der Jahrestagung des Deutschen Ethikrats wurde in einem Forum unter der Leitung von Michael Wunder folgende These herausgearbeitet: Es bestünden zwar viele positive Datenanwendungen für Lifelogging und Selbstvermessung, z.B. in Form von Mensch-Maschine-Nutzungen bei der Alters- und Pflegeassistenz. Die Geräte zur Datenmessung oder -erhebung würden aber Mängel in 
Bezug auf die Datenqualität und die Zuverlässigkeit aufweisen. Zudem hätten gerade Menschen, die auf solche Geräte angewiesen sind, grosses Misstrauen gegenüber der Weitergabe bzw. der Speicherung der Daten. ${ }^{27}$ Um dieses Misstrauen abzubauen, „müsste also viel mehr auf Transparenz, Kontrollierbarkeit und Hoheit über die eigenen Daten geachtet werden, auf Nutzerorientierung in der Herstellung und im Angebot dieser Geräte" (Wunder, Deutscher Ethikrat, 2015, S. 118).

\subsubsection{Selbstbestimmung}

Die Beachtung der Selbstbestimmung des Menschen ist Inhalt des zentralen medizinethischen Prinzips „Respect for Autonomy“ (Beauchamp \& Childress, 2001). Härle (2011) beschreibt die Selbstbestimmung als Recht eines Menschen „wohlüberlegt selbst darüber zu entscheiden, ob eine mögliche Therapie an ihm durchgeführt werden soll oder nicht" (S. 273).

Dabrock beschreibt Selbstbestimmung nach dem Verständnis von Volker Gerhardt als "das Praktisch-Werden von Freiheit am Orte des Individuums, das in soziale Beziehungen in hoffentlich möglichst gerechten Institutionen eingebunden bleibt" (Dabrock, Deutscher Ethikrat, 2015, S. 26). Diese Idealsituation stellt Christiane Woopen (Deutscher Ethikrat, 2015) in der gegenwärtigen Entwicklung von personalisierter Werbung infrage: Entpuppt sich das, was wir als Selbstbestimmung erkennen, am Ende möglicherweise als Selbstentmündigung? Lassen wir uns unsere Umgebung von unbekannten anderen und auch der Werbeindustrie gestalten, anstatt sie selbst zu gestalten?

Weitere Risiken betreffend Selbstbestimmung und Autonomie bestehen in den sich regelmässig ändernden Inhalten von allgemeinen Geschäftsbedingungen (AGB). Angesichts dieser Problematik werfen Fangerau, Grimmert und Albrecht (2016, S. 205) die Frage auf, „inwiefern eine Person ihre Einwilligung überhaupt in Klauseln geben kann, die zum einen erst zu einem zukünttigen Zeitpunkt erstellt werden, zum anderen gleichzeitig jedoch auch rückwirkend in Form der angesammelten Datenmenge zum Tragen kommen und möglicherweise weitreichende Konsequenzen für die Nutzerin oder den Nutzer haben, die zum Einwilligungszeitpunkt in keiner Weise absehbar sind".

27 Es stellt sich für sie insbesondere die Frage, wie die Daten weitergegeben bzw. wo sie gespeichert werden (siehe Kapitel 7.3). 
Die Selbstbestimmung Einzelner bzw. ihre Autonomie wird durch die Anwendung von Big Data eingeschränkt. Für Thomas Petri (Deutscher Ethikrat, 2015) steht Big Data auch für eine philosophische Haltung, die nach maximalem Erkenntnisgewinn strebt, indem alle verfügbaren Daten gesammelt werden, um eine möglichst uneingeschränkte Nutzung der Daten zu gewährleisten. Petri hält denn auch fest, dass man mit Big Data „den Nachweis von Kausalitätsverhältnissen durch statistische Korrelationen ersetzt" habe (Petri, Deutscher Ethikrat, 2015, S. 29). Dabrock (Deutscher Ethikrat, 2015) führt an, dass man zwar Korrelationsanalysen benötige, doch könne es zu problematischen Entwicklungen führen, „wenn Korrelationen als Kausalitäten ausgegeben oder als solche wahrgenommen werden". Dabei würden Dinge zusammenwachsen, die nicht zusammengehören (Dabrock, Deutscher Ethikrat, 2015, S. 27). Auch Hauser et al. (2017) sehen die Autonomie des Individuums durch statistische Korrelationen tangiert. Statistischen Korrelationen würden auf Grundlage sämtlicher Kundinnen und Kunden generiert und anschliessend auf eine Einzelperson übertragen werden. Dabei treffe das auf der Gesamtheit aller Kundinnen und Kunden basierende Muster nicht zwingend auf jedes Individuum zu (Hauser et al., 2017). Dies kann auch zur Diskriminierung von Personen führen (vgl. 10.3.4).

Selke (2016, S. 332) stellt in Anlehnung an Rauner und Schröder (2015) die Frage, „was passiert, wenn Maschinen (bzw. Algorithmen) nicht nur simple Entscheidungen beeinflussen, sondern auch komplexe Entscheidungen übernehmen. Für das Leben in der Perfektionskolonie werden immer raffiniertere smarte Helfer entwickelt. Dabei stellt sich die Frage, wann die Maschinen uns erstmals auch ethische Entscheidungen, z.B. solche über Leben und Tod abnehmen werden."

\subsubsection{Gleichheit und Nichtdiskriminierung}

Eine Diskriminierung liegt vor, wenn eine Person ungleich behandelt wird allein aufgrund ihrer Zugehörigkeit zu einer bestimmten Gruppe, welche historisch oder in der gegenwärtigen sozialen Wirklichkeit tendenziell ausgegrenzt oder als minderwertig angesehen wird. Die Diskriminierung stellt eine qualifizierte Ungleichbehandlung von Personen in vergleichbaren Situationen dar, indem sie eine Benachteiligung von Menschen bewirkt, die als Herabwürdigung oder Ausgrenzung einzustufen ist, weil sie an Unterscheidungsmerkmalen anknüpft, die einen wesentlichen und nicht oder nur schwer aufgebbaren Bestandteil der Identität der betroffenen Personen ausmachen. Insofern beschlägt das Diskriminierungsverbot 
auch Aspekte der Menschenwürde (nach regelmässiger bundesgerichtlicher Rechtsprechung, beispielsweise BGE $139 \mid$ 169, E. 7.2.1).

Nichtdiskriminierung gehört auch zu den Fairnessgeboten, die in unserer Gesellschaft unbestritten sind. Dass Menschen nicht immer gleichbehandelt werden, wird allgemein akzeptiert. Gemäss Hauser et al. (2017) wird eine Ungleichbehandlung ethisch problematisch, wenn dabei Kriterien massgebend sind, die im Hinblick auf den Zugang zu bestimmten Gütern, Chancen und Positionen nicht relevant seien.

Immer wieder wird in der Literatur die Einbindung von Fitness- bzw. GesundheitsApps in Prämienprogramme von Versicherungen diskutiert. Werden fitnessbewusste Personen, die ihre Gesundheitsdaten dokumentieren und den Versicherungen zur Verfügung stellen, durch niedrigere Prämien belohnt, bedeutet dies, dass Menschen, die diese Daten nicht teilen wollen oder können, höhere Prämien bezahlen und damit ungleich behandelt werden. Fangerau, Grimmert \& Albrecht (2016) nennen als weiteres Beispiel die Firma dacadoo. Diese spricht Unternehmen an, ihre App als "Gesundheitslösung in Eigenmarke“, ${ }^{28}$.d.h. als innerbetriebliche Fitness-App, zu implementieren. Fraglich wird dadurch gemäss Fangerau, Grimmert \& Albrecht (2016, S. 206) die Sicherstellung der „wirklich freiwilligen Teilnahme an betrieblich organisierten und überwachbaren Gesundheitsprogrammen".

Einen interessanten Gesichtspunkt bringt Brigitte Tag (persönliches Interview, 21.9.2016) ein, wenn sie festhält, dass eHealth eine Art QS darstelle, weil die Patientinnen und Patienten die Möglichkeit erhalten, den Inhalt des eigenen elektronischen Patientendossiers mitzubestimmen. Was aber, wenn eine Patientin oder ein Patient diese Mitbestimmung über die Informationen ablehnt, weil eine Information zu einer Diskriminierung führen könnte? Einen weiteren Aspekt heben die Autoren des White Papers „Big Data im Gesundheitswesen“ (Fischer et al., 2015) hervor. Die Deanonymisierung von Daten berge das Risiko, Einzelpersonen zu diskriminieren, beispielsweise durch Arbeitgeber, Versicherungen oder andere Akteure. Béranger (2015, S. 14) führt hierzu Folgendes an: „Big Data risks magnifying the phenomenon of categorization, classification, discrimination and their byproducts within society, as the data become more and more closely associated with the person." Des Weiteren identifizieren Fangerau, Grimmert \& Albrecht (2016) eine soziale Ungleichbehandlung, wenn der Zugang zu medizinischer

$28 \mathrm{https}$ ://info.dacadoo.com/de/unternehmenslosungen/firmenlosungen/ (abgerufen am 16.10.2016). 
Technik wegen hoher Kosten für eine Minderheit verschlossen bleibt und dies zu Krankheit oder gar Tod führt.

Selke (Deutscher Ethikrat, 2015) hat im Kontext von Lifelogging den Begriff der rationalen Diskriminierung zur Diskussion gebracht (vgl. Kapitel 4.1.1 und 4.1.3). Nach Selke bedeutet rationale Diskriminierung, dass Menschen in der Gesellschaft nicht mehr aufgrund von „sexistischen, rassistischen, ethnischen, ethnozentristischen“ oder ähnlichen Motiven diskriminiert werden, „sondern [basierend] auf vermeintlich rationalen wissenschaftlichen, proto-wissenschaftlichen, scheinbar objektiven Messverfahren" (S. 58). Gemäss Selke (Deutscher Ethikrat, 2015) verändert die Technologie des Lifelogging innerhalb kurzer Zeit das Wertegefüge sowie die Kultur unserer Gesellschaft, indem neue strukturelle Bedingungen für soziale Abwertung geschaffen werden. Er nennt dazu drei Praxisbeispiele:

- Arbeitsplatz: Betriebliches Gesundheitsmanagement fungiert als Deckmantel für Konformismus und Solidaritätsbeschneidung. Autonome Entscheidungen werden durch soziale Kontrolle ersetzt.

- Versicherungen: Versicherungsleistungen werden auf der Basis von Risikoäquivalenzprinzipien durch zuvor berechnete Wahrscheinlichkeiten vergeben. Dadurch werden Leistungen und Vergünstigungen nur noch ermöglicht, wenn individuelle Daten zur Verfügung gestellt werden.29

- Technische Assistenzsysteme: Diese Systeme werden in der Pflege älterer Menschen eingesetzt, beispielsweise durch die Ausstattung von Wohnräumen mit Sensoren. Selke nennt dies die „Assistive Kolonialisierung“. Das Eindringen von Assistenzsystemen in den privaten Lebensraum der Gepflegten werde das, was wir für Fürsorge halten, verändern und Werte verschieben. Die Frage ist: Wer definiert, was normal ist in diesen mit Sensoren ausgestatteten Wohnräumen, und wer definiert, wann ein Alarm ausgelöst wird? Der Mensch oder ein Sensor?

Selkes These (Deutscher Ethikrat, 2015, S. 59) lautet: „Rationale Diskriminierung verändert schleichend die Bewertung von Menschen und erzeugt ethische Pro-

29 Beispiel: Die AXA Winterthur belohnt Personen unter 26 Jahren, die einen Drive Recorder installieren und ihr Fahrverhalten aufzeichnen lassen, mit bis zu 25\% Rabatt, gemäss http://www.tagesanzeiger.ch/auto/technik/Wir-sehen-die-Zukunft-beim-DriveRecorder/ story/14512230 (abgerufen am 16.10.2016). 
bleme da, wo es um existenzielle Entscheidungen geht. Wenn Menschen zu Zahlenkörpern werden, weil Daten soziale Erwartungen übersetzen, kann mit den Menschen viel rücksichtsloser und gleichgültiger verfahren werden. Darin liegt das ethische Problem. Wenn durch Quantifizierung die persönlichen Umrisse von Menschen verschwinden, bedeutet das auf lange Sicht die Ausschaltung einer differenzierten Persönlichkeit, einer pluralen Gesellschaft, und es etabliert sich ein Menschenbild, das funktionalistisch ist."

\subsubsection{Informationelle Selbstbestimmung und Kontrolle der eigenen (digitalen) Identität}

Mittels der informationellen Selbstbestimmung soll eine Person die Kontrolle darüber ausüben können, „was mit sie betreffenden Informationen geschieht; sie sollen vor Benachteiligungen durch die Verwendung der Daten geschützt werden" (Brunner, 2003, S. 46). Die Forderung nach informationeller Selbstbestimmung ist eng verbunden mit der Forderung nach Kontrolle über die eigene digitale Identität. Digitale Identitäten werden durch Big-Data-Anwendungen konstruiert. So sagen einzelne Daten, beispielsweise das Tippverhalten, noch nichts über die finanzielle Bonität einer Person aus. Wenn jedoch eine grosse Anzahl solcher einzelnen Daten miteinander kombiniert werden, entsteht eine neue digitale Identität, die Rückschlüsse auf die Bonität einer Person zulassen. Solche Rückschlüsse werden ethisch problematisch, wenn Kundinnen und Kunden nichts davon wissen und auch keine Möglichkeit haben, diese Fehlschlüsse zu korrigieren (Hauser et al., 2017). Ein weiteres Problem könnte durch die Anwendung von Big-Data-Technologien entstehen, wenn diese Technologien zu einer permanenten kommerziellen Überwachung durch Unternehmen führen und den Anwenderinnen und Anwender damit die Freiräume für nicht strategisches Verhalten entziehen (Hauser et al., 2017).

Eine negative Folge der Ausübung des Rechts auf informationelle Selbstbestimmung kann darin bestehen, dass Versicherte eine Versicherungsleistung nicht erhalten oder einen wesentlich höheren Preis dafür bezahlen müssen, weil sie sich weigern, private Informationen preiszugeben. Benötigt eine Versicherung jedoch private Informationen, um beispielsweise das Risikomanagement zu verbessern oder Prämien zu berechnen, braucht es eine Abwägung zwischen dem Wert der informationellen Selbstbestimmung und den Ansprüchen der Versicherung (Hau- 
ser et al., 2017). Die eben zitierten Autorinnen und Autoren sehen es ethisch jedoch als nicht problematisch an, wenn Nutzende ihre digitalen Identitäten selektiv und einseitig verwenden und somit keine vollständige digitale Identität preisgeben, sofern natürlich keine Betrugsabsichten bestehen (Hauser et al., 2017).

\subsubsection{Solidarität}

In der deutschsprachigen Literatur wird oft die Frage diskutiert, ob Versicherungen und speziell Krankenversicherungen gegen das Solidaritätsprinzip verstossen, wenn sie Versicherten Rabatte gewähren, die bereit sind, ihre Selbstvermessungsdaten via Gesundheits-Apps zur Verfügung stellen (Fangerau, Grimmert \& Albrecht, 2016). In der Schweiz gilt das Solidaritätsprinzip für die Grundversicherung, dabei dürfen Prämien grundsätzlich nicht nach Krankheitsrisiko abgestuft werden (Gächter \& Rütsche, 2013). Diese Diskussionen werden in der Schweiz zwar geführt (Tag, persönliches Interview, 21.9.2016), sie sind jedoch noch nicht in gleicher Weise relevant wie in Deutschland (zur Diskussion in Deutschland, Fangerau, Grimmert \& Albrecht, 2016).

Im Privatversicherungsbereich geht es hingegen um Privatautonomie, Vertragsfreiheit sowie um das Versicherungsprinzip und damit auch um die Frage der Risiken, die von einer Mehrzahl von Versicherten getragen werden (Baumann, 2008). Damit haben Versicherungen die Möglichkeit, Personen, die sich zu wenig bewegen, vom Privatversicherungsschutz auszuschliessen oder höhere Prämien zu verlangen. Dies ist auch bei Personen mit Prädispositionen möglich - und könnte gemäss Brigitte Tag ein ethisches Problem darstellen. Grundsätzlich sind diese Personen jedoch durch den allgemein gut ausgebauten Leistungskatalog der Grundversicherung abgedeckt und somit in der Grundversicherung aufgehoben (Tag, persönliches Interview, 21.9.2016).

Nach Hauser et al. (2017) können im Rahmen von Big-Data-Anwendungen verschiedene Daten über das persönliche Verhalten zwecks individueller Risikobeurteilung kombiniert werden. Die so erstellten individuellen Risikoprofile könnten im Anschluss als Ausdruck eines selbstbestimmt gewählten Verhaltens klassifiziert und als ethisch legitimierter Grund zum Ausschluss grosser Risiken herangezogen werden. Letztlich würde man damit die Solidarität untergraben (Hauser et al., 2017). Wenn Versicherungen von ihren Kundinnen und Kunden schliesslich ver- 
langen, mittels Selbstvermessung die Einhaltung bestimmter Ernährungs- und Fitnessgewohnheiten zu überprüfen, führt dies zusätzlich zu einem Konflikt mit dem Recht auf Selbstbestimmung (Hauser et al., 2017).

Brigitte Tag (persönliches Interview, 21.9.2016) spricht weitere Ungerechtigkeiten eines solchen Systems an: Was, wenn Personen, die an einer Gehbehinderung leiden, den Versicherungen ihre Bewegungsdaten zur Verfügung stellen? Wie geht man mit diesen Menschen um? Müsste hier nicht ein Ausgleich geschaffen werden? Ähnliche Diskussionen werden schon seit Jahrzehnten geführt, beispielsweise in Verbindung mit der Ausübung von Risikosportarten. Wer Risikosportarten betreibt, muss im Schadensfall mit gewissen Restriktionen rechnen. Sind spezifische Risiken nicht versichert, fragt es sich aber, wer im Schadensfall dafür aufkommen muss. Meist dürfte in Fällen, in denen jemand wirklich Hilfe benötigt, die Allgemeinheit für den Schaden aufkommen (Subsidiarität des Staates). Es stellt sich dann jedoch die Frage, ob so nicht "Lasten auf die Allgemeinheit verschoben werden, die so nicht verschoben werden sollten" (Tag, persönliches Interview, 21.9.2016).

\subsection{Fazit ethische Aspekte}

Manche Autorinnen und Autoren sehen in der QS-Datenflut einen Innovationstreiber. Es wird die Meinung vertreten, dass die Nutzung dieser Daten letztlich zur Steigerung der Lebensqualität beitrage. Durch die Kombination verschiedener Daten sollen Erkenntnisse gewonnen werden, die sowohl den einzelnen Anwenderinnen und Anwendern als auch der gesamten Bevölkerung zugutekommen und die Gesellschaft als Ganzes effizienter machen. Demgegenüber heben andere Autorinnen und Autoren die ethischen Herausforderungen bzw. Risiken für Werte wie Privatheit, Transparenz, (informationelle) Selbstbestimmung, Gleichheit und Solidarität hervor.

Diverse Studien verweisen auf ethische Herausforderungen im Umgang mit QS und thematisieren vornehmlich damit verbundene Risiken. Einzelne Studien weisen jedoch darauf hin, dass es durchaus auch Anwendungschancen gebe. Die Abwägung der mitunter konkurrierenden Interessen und Werte kann unter Umständen zu (normativen) Zielkonflikten und moralischen Dilemmasituationen führen, die oft nur subjektive Lösungsansätze zulassen. In diesen Fällen bedarf es 
einer eingehenden sozialethischen Debatte und als ultima ratio einer gesetzgeberischen Lösung.

Peter Dabrock vom Deutschen Ethikrat (2015, S. 26) findet in diesem Zusammenhang klare Worte: „[W]ill man diesem Trend [der Vermessung durch Big-DataAnwendungen] verantwortlich begegnen, muss die Zivilgesellschaft und die sie repräsentierende Politik versuchen, das Heft des Handelns wieder zurückzugewinnen. Das funktioniert nur - und es ist höchste Zeit, dass das auf hoher Ebene geschieht-, wenn Rahmenordnungen aufgesetzt werden, die gesellschaftlich verantwortbar sind." 


\section{www.ta-swiss.ch}

Preiswerte kleine Sensoren in Tracking-Geräten und Smartphones machen es möglich: die ständige Überwachung der Fitness, Wellness oder Gesundheit. "Quantified Self", das Messen der eigenen Körper- und Verbaltensaktivitäten, findet immer mehr Verbreitung. Anwenderinnen und Anwender nutzen Quantified Self vor allem, um ibre körperlichen Aktivitäten zu optimieren. Die Daten sind aber auch für die Forschung von Interesse: Aus der Datenfülle sollen Erkenntnisse gewonnen werden für die Früherkennung von Krankheiten und für verbesserte Therapien. Zudem wollen Akteure aus der Wirtschaft und dem Gesundheitsbereich von den Auswertungen profitieren. Der Umgang mit den heiklen Angaben zur Gesundheit muss dabei den gesetzlichen Anforderungen des Datenschutzes genügen.

Die interdisziplinäre Studie untersucht Chancen und Risiken des Quantified Self. Sie zeigt auf, welche Geräte und Dienstleistungen es gibt und welche künftigen Entwicklungen zu erwarten sind. Neben medizinischen und technischen Aspekten werden auch gesellschaftliche, ethische, rechtliche und ökonomische Fragen analysiert und Empfehlungen formuliert.

\section{V/llf}

TA-SWISS 67/2018

ISBN 978-3-7281-3891-0

(Printausgabe)

ISBN 978-3-7281-3892-7

(E-Book)

DOI 10.3218/3892-7
Schweizerische Eidgenossenschaft

Confédération suisse

Confederazione Svizzer

Confederaziun svizra

Eidgenössisches Departement des Innern EDI

Bundesamt für Gesundheit BAG

\section{ehealthsuisse}

Kompetenz- und Koordinationsstelle

von Bund und Kantonen

Centre de compétences et de coordination de la Confédération et des cantons

Centro di competenza e di coordinamento di Confederazione e Cantoni 